Department of Applied Mathematics

Faculty of EEMCS

University of Twente
The Netherlands
P.O. Box 217

7500 AE Enschede

The Netherlands

Phone: +31-53-4893400

Fax: $+31-53-4893114$

Email: memo@math.utwente.nl

www.math.utwente.nl/publications

Memorandum No. 1792

Forecasting market impact costs and

identifying expensive trades

J.A. BiKKER ${ }^{1}$, L. SPIERdiJK,

R.P.M.M. HoEvenaARs ${ }^{2}$ AND P.J. VAN DER SLuis ${ }^{3}$

February, 2006

ISSN 0169-2690

\footnotetext{
${ }^{1}$ Supervisory Policy Division, Strategy Department, De Nederlandsche Bank, P.O. Box 98, NL-1000AB Amsterdam

${ }^{2}$ Maastricht University, Dept. of Quantitative Economics, Financial and Risk Policy Department, ABP, P.O. Box 75753, NL-1118ZX Schiphol

${ }^{3}$ Vrije Universiteit Amsterdam, Dept. of Finance and Financial Sector Management, GTAA Fund, ABP Investments, P.O. Box 75753, NL-1118ZX Schiphol
} 


\title{
Forecasting Market Impact Costs and Identifying Expensive Trades
}

\author{
Jacob A. Bikker \\ Senior Economist \\ Supervisory Policy Division \\ Strategy Department \\ De Nederlandsche Bank \\ P.O. Box 98 \\ NL-1000AB Amsterdam \\ The Netherlands \\ Phone: +3120 5242352 \\ j.a.bikker@dnb.nl \\ Laura Spierdijk \\ Assistant Professor \\ Financial Engineering Lab, University of Twente \\ P.O. Box 217 \\ NL-7500AE Enschede \\ The Netherlands \\ Phone: +31 534893386 \\ l.spierdijk@utwente.nl \\ Roy P.M.M. Hoevenaars \\ Senior Researcher ALM \\ Maastricht University \\ Department of Quantitative Economics; \\ Financial and Risk Policy Department, ABP \\ P.O. Box 75753 \\ NL-1118ZX Schiphol \\ The Netherlands \\ Phone: +31 204059827 \\ roy.hoevenaars@abp.nl \\ Pieter Jelle van der Sluis \\ Senior Portfolio Manager \\ Vrije Universiteit Amsterdam \\ Department of Finance and Financial Sector Management; \\ GTAA Fund, ABP Investments \\ P.O. Box 75753 \\ NL-1118ZX Schiphol \\ The Netherlands \\ Phone: +3120405 4343 \\ pieterjelle.vander.sluis@Abpinvestments.nl
}




\begin{abstract}
Often, a relatively small group of trades causes the major part of the trading costs on an investment portfolio. For the equity trades studied in this paper, executed by the world's second largest pension fund, we find that only $10 \%$ of all trades determines $75 \%$ of total market impact costs. Consequently, a reduction of the trading costs of comparatively few expensive trades would already result in substantial savings on total trading costs. Since trading costs depend to some extent on controllable variables, investors can try to reduce trading costs by carefully controlling these factors. As a first step in this direction, this paper focuses on the identification of expensive trades before actual trading takes place. However, forecasting market impact costs appears notoriously difficult and traditional methods fail. Therefore, we propose two alternative methods to form expectations about future trading costs. The first method uses five 'buckets' to classify expensive trades, where the buckets represent increasing levels of market impact costs. Each trade is assigned to a bucket depending on the probability that the trade will incur high market impact costs. The second method identifies expensive trades by considering the probability that market impact costs will exceed a critical level. When this probability is high, a trade is classified as potentially expensive. Applied to the pension fund data, both methods succeed in filtering out a reasonable number of trades with high trading costs. The results underline the productive role that model-based forecasts can play in trading cost management.
\end{abstract}

Keywords: market impact costs, forecasting, institutional trading, trading cost management

AMS Subject Classification: 62M20, 91B28, 91B84 
It is a well-known phenomenon that trading costs can substantially reduce portfolio performance. A stock with a high gross return may end up with a relatively low net return when trading costs are high. Therefore, trading costs are an important factor to consider when portfolio decisions are made.

There is a vast literature on trading costs and their determinants; see Keim and Madhavan [1998] for an excellent survey. Usually, the literature distinguishes explicit and implicit trading costs. The explicit part consists of fixed costs, such as commissions, taxes, and fees. Implicit costs are more variable and are built up of market impact costs (price impact), bid-ask spread, delay costs (the costs of adverse price movements that may occur when trading is postponed), and opportunity costs (the costs of not trading). Market impact costs are usually found to be the most important component of trading costs and occur when price effects cause execution prices to be less favorable than benchmark prices.

Often, a comparatively small group of trades causes the major part of market impact costs. For the equity trades studied in this paper, executed by the world's second largest pension fund ABP, we find that only $10 \%$ of all trades determines $75 \%$ of total market impact costs. Consequently, reducing the trading costs of relatively expensive trades would already lead to substantial savings on total trading costs. Since trading costs depend to some extent on controllable factors such as broker intermediation, investment style, trade timing, and trading venue (see e.g. Bikker, Spierdijk, and Van der Sluis [2005]), investors can try to reduce trading costs by carefully controlling these factors. For instance, when forecasted trading costs exceeds a certain critical level, investors may decide to choose e.g. another type of broker intermediation, trade timing or trading venue or to monitor the trade more closely during execution.

Since model-based forecasts of market impact costs can contribute to identifying potentially expensive trades, they play a crucial role in transaction costs management. Although the importance of forecasting trading costs has been widely recognized (see e.g. Cheng [2003] and Konstance [2003]), the literature has paid surprisingly little attention to this issue. To fill the gap, this paper proposes two alternative methods to identify expensive trades before actual trading takes place. Furthermore, we also discuss how to incorporate the proposed forecasting methods in the stock selection process. We illustrate the approach with a unique data set covering all global equity trades in the first quarter of 2002 executed by the world's second largest pension fund.

\section{DATA AND DEFINITION}

The 'Algemeen Burgerlijk Pensioenfonds' (ABP) is the largest Dutch pension fund and second largest of the world. It has about 2.6 million clients and an invested 
capital of approximately 190 billion Euro ${ }^{1}$, corresponding to one third of total Dutch pension fund assets. The data set contains detailed information on all 3,721 worldwide internally managed equity trades of ten different funds at ABP during the first quarter of 2002, with a total transaction value of 5.7 billion Euro. Of these trades, 1,962 are buys and 1,759 are sells executed in Europe, the United States, Canada, and Japan. The trades in this sample consist of transactions for risk control and rebalancing of index trackers, as well as informed ones for active management. The internally managed equity portfolios in our sample have a total value of about 20 billion Euro.

For each transaction the data set provides the execution price and the price of the stock just before the trade was passed to the broker. Moreover, the data also specify when the trade was submitted to the broker and when it was executed. Additionally, the data include detailed information on several trade, exchange, and stock specific characteristics, including momentum, relative trade size, market capitalization, type of broker intermediation, investment style, trade timing, and trading venue. The first two columns of Exhibit 3 provide a complete list of the variable names and their definitions.

We notice that we have constructed the data set on the basis of the post-trade analysis provided by ABP; the remaining data come from Factset and Reuters. For more details on the data, we refer to Bikker, Spierdijk and Van der Sluis [2005].

\section{PRELIMINARY DATA ANALYSIS}

Market impact costs occur when the execution price of a trade is worse than the benchmark price. Hence, in order to forecasts these costs, a benchmark price has to be chosen. We opt for the pre-execution benchmark, in line with e.g. Wagner and Edwards [1993]. More precisely, we take as the benchmark the price at the moment the order was passed to the broker and correct for market-wide price movements during the trade, as in Chan and Lakonishok [1995, 1997]. The MSCI World industry group indices are used as a proxy for these market movements. Thus, for a buy transaction in stock $i$ at time $t$ market impact costs $\left(\mathbf{C}_{i t}^{B}\right)$ are defined as

$$
\mathbf{C}_{i t}^{B}=\underbrace{\log \left(\mathbf{P}_{i t}^{\text {exe }} / \mathbf{P}_{i t}^{p t}\right)}_{\text {price impact }}-\underbrace{\log \left(\mathbf{M}_{i t}^{\text {exe }} / \mathbf{M}_{i t}^{p t}\right)}_{\text {market wide price movements }},
$$

where $\mathbf{P}_{i t}^{e x e}$ and $\mathbf{P}_{i t}^{p t}$ denote the execution and pre-trade price of stock $i$ at time $t$, respectively. $\mathbf{M}_{i t}^{e x e}$ and $\mathbf{M}_{i t}^{p t}$ denote the value of the MSCI industry group index corresponding to stock $i$ at the time of the execution of the trade and at the pre-trade time, respectively. In a similar way we define market impact costs of sells:

$$
\mathbf{C}_{i t}^{S}=\log \left(\mathbf{P}_{i t}^{p t} / \mathbf{P}_{i t}^{e x e}\right)-\log \left(\mathbf{M}_{i t}^{p t} / \mathbf{M}_{i t}^{e x e}\right) .
$$

\footnotetext{
${ }^{1}$ This is the total invested capital at the end of 2005.
} 
For both buys and sells, positive market impact cost indicate that a trade has been executed against a price worse than at the moment of trade initiation.

To get a first impression of the magnitude of trading costs, we calculate principalweighted average market impact costs. To obtain the principal-weighted statistics we weight each observation by the Euro value of the trade, so that larger trades contribute more than smaller ones. Average market impact costs of buys equal 20 basis points (bp) with a standard deviation of $6 \mathrm{bp}$ and those of sells $30 \mathrm{bp}$ with a standard deviation of $7 \mathrm{bp}$. Including commissions, these costs equal $27 \mathrm{bp}$ for buys and $38 \mathrm{bp}$ for sells, with respective standard deviations of $6 \mathrm{bp}$ and $7 \mathrm{bp}$. These price effects are relatively moderate compared to other studies (see Bikker, Spierdijk, and Van der Sluis [2005]).

Exhibit 1 depicts the contribution of each trade to total market impact costs. Starting with all trades sorted from cheap to expensive, the horizontal axis denotes the percentage of trades executed (in the range 0-100\%). The vertical axis represents the total trading costs (including commission) in Euro corresponding to the trades executed. We see that the $25 \%$ cheapest trades have negative market impact costs, whereas the $35 \%$ most expensive trades incur positive trading costs. The remaining $40 \%$ of medium-expensive trades have market impact costs close to zero. Together, they yield a convex and asymmetric 'market impact costs smile'. The convexity in Exhibit 1 implies that the $10 \%$ most expensive trades cause about $75 \%$ of total market impact costs. Consequently, the investor could already realize substantial savings on total trading costs if he or she would be able to (1) identify a few expensive trades before actual trading and (2) reduce the trading costs incurred on these trades, e.g. by more careful monitoring.

We make this more explicit by means of simulation. For this purpose, we consider an investor that has a certain skill (between 0-100\%) to identify expensive trades. When an investor has a skill of $100 \%$ he or she is able to rank all trades correctly according to future trading costs ('perfect foresight'); with a $0 \%$ skill, the investor's ranking of the stocks is completely random. ${ }^{2}$ At the same time we consider a cost reduction percentage that applies to the selected trades. For all skill levels and each percentage of cost reduction per trade, we simulate the corresponding total realized savings on trading costs. ${ }^{3}$ The resulting 3 -dimensional graph in Exhibit 2 (a) displays the relation between investor skill ( $x$-axis), cost reduction per trade ( $y$-axis), and total expected savings ( $z$-axis). For instance, an investor skill of $20 \%$ and a cost reduction of $20 \%$ per trade results in expected savings of almost 1.4 million Euro.

Although the extremes of no and $100 \%$ skill are quite trivial, the simulation reveals various nontrivial patterns in Exhibit 2 (a). The contour plot in Exhibit 2 (b)

\footnotetext{
${ }^{2}$ For any skill of $p \%$ with $0<p<1, p \%$ of all trades is ranked correctly and the other $(1-p) \%$ is ranked randomly over the remaining positions.

${ }^{3}$ We repeat this 1,000 times and average the realized savings over the simulation runs to obtain the expected savings for each skill level and cost reduction percentage.
} 
(where each contour line represents an additional saving of 5 million Euro) highlights the nonlinear relation between trading costs and cost reduction. Moving from south-west to north-east in Exhibit 2 (b), the distances between the contour lines get smaller. Saving an additional 5 million Euro requires a comparatively large improvement in either skill or cost reduction whenever these are low. By contrast, saving another 5 million needs to be combined with a much smaller improvement once these are already high. Also, when investor skills are low and the percentage of cost reduction is high (point $A$ ), relatively more cost reduction than skill improvement is needed to arrive at lower market impact costs. Similarly, with high investor skills and low cost reduction (point $B$ ), relatively more skill improvement than reduction is required to reduce market impact costs. Also, the contour lines show that relatively low skill values in combination with a substantial cost reduction per trade result in the same savings on total trading costs as relatively high skill values and low cost reduction percentages.

\section{DETERMINANTS OF MARKET IMPACT COSTS}

Market impact costs usually depend on various trade, exchange, and stock specific characteristics. To formalize this, we assume that the market impact costs of a buy trade are determined by $N$ factors (say $\mathbf{X}_{1}, \ldots, \mathbf{X}_{N}$ ) and a random noise term $\varepsilon$. Since trading costs of buys and sells usually show different behavior, we follow the literature and consider separate models for them. Without loss of generality, we confine ourselves here and in the sequel to buy trades. We deal in exactly the same way with sell trades, using similar notation. Thus, we assume that market impact costs of buy trades $\left(\mathbf{C}^{B}\right)$ satisfy

$$
\mathbf{C}^{B}=\beta_{0}^{B}+\sum_{j=1}^{N} \beta_{j}^{B} \mathbf{X}_{j}+\varepsilon^{B} .
$$

For the $N$ factors we take the variables described before (see also Exhibit 3 ). We estimate the resulting linear regression model using least squares. Exhibit 3 displays the corresponding estimated coefficients and $R^{2}$ 's. For a detailed interpretation of the model coefficients, we refer to Bikker, Spierdijk, Van der Sluis [2005].

\section{FORECASTING MARKET IMPACT COSTS}

The model in equation (3) explains market impact costs of buy and sell trades from various, to some extent controllable, factors. In this section we use the model to forecast future market impact costs. The resulting forecasts can play a useful role in transaction cost management. For instance, when forecasted trading costs exceed a certain critical level, the investor may decide to choose another type of broker intermediation, or trade timing, trading venue, or to monitor the trade more closely during execution.

Throughout, we evaluate the forecasting power of the model both in-sample and

out-of-sample. To do so, we divide the data sample into an in-sample part (the first 
two months of trades, about $75 \%$ of the total sample, say trades $t=1, \ldots, n)$ and an out-of-sample part (the final month of trades corresponding to $25 \%$ of the sample, say trades $t=n+1, \ldots, n+m)$. Subsequently, we estimate the models for buy and sell trades using only the in-sample data. The in-sample forecasts correspond to the predicted trading costs for the in-sample trades. For the out-of-sample forecasts we proceed in a different way. We start with estimating the model using trades $t=1, \ldots, n$. Next, we obtain a forecast for trade $t=n+1$. In the subsequent steps we add one trade at a time and use an expanding window estimator to re-estimate the models using all data available up to the day preceding trade $t$. Subsequently, we calculate forecasts for one trade ahead, i.e. for trade $t+1$. We repeat this step-wise for each trade $t=n+1, \ldots, n+m$.

\section{Numeric forecasts of market impact costs}

There are several ways to forecast future market impact costs. For the moment we assume that the investor's goal is to predict the trading costs of every trade to be executed. In this case, the investor needs a numeric forecast of his or her market impact costs. The common way to forecast market impact costs is by means of expected market impact costs. Assuming that the noise term in specification (3) has mean zero, expected trading costs of buys equal

$$
\mathbb{E}\left(\mathbf{C}^{B}\right)=\beta_{0}^{B}+\sum_{j=1}^{N} \beta_{j}^{B} \mathbf{X}_{j}
$$

Given estimates of the coefficients $\beta_{j}^{B}$ based on historical data, we easily calculate expected trading costs conditional on factors $\mathbf{X}_{1}, \ldots, \mathbf{X}_{N}$ by means of formula (4).

Exhibits 4 (a)-(d) display scatter diagrams of realized costs and forecasted trading costs, together with regression lines that capture the relation between forecasted and realized costs. Clearly, forecasted trading costs differ substantially from realized costs. This is confirmed by formal error measures, such as the mean average percentage error. Since market impact costs reflect the price movements of a stock during trade execution, the difficulty of forecasting these costs does not come as a complete surprise. Moreover, the out-of-sample period differs substantially from the in-sample months, which also complicates forecasting. ${ }^{4}$ Nevertheless, even for this turbulent period the upward slopes of the regression lines in Exhibit 4 reflect fairly positive correlations between realized and forecasted trading costs. In-sample, the correlations equal 0.44 for buys and 0.50 for sells. Out-of-sample they take the values 0.21 and 0.19, respectively. All correlations are significant at a $5 \%$ significant level. Hence, the model forecasts higher trading costs for stocks that actually experience higher trading costs.

\footnotetext{
${ }^{4}$ In the year 2002, January was bearish and February was quite flat. However, the out-of-sample month of March was bullish.
} 


\section{Bucket classification approach}

Although the forecast quality of the model in equation (3) is limited, the model forecasts higher trading costs for stocks that actually experience higher trading costs. Therefore, rather than providing numeric forecasts of market impact costs, we expect to be more successful in the classification of market impact costs in terms of 'high' or 'low'. Moreover, we have seen that only $10 \%$ of all trades determines $75 \%$ of total market impact costs. This emphasizes that, from the perspective of cost reduction, the focus should be on detecting the most expensive trades.

To predict trading costs in terms of 'high' or 'low', we distinguish five buckets with predefined boundaries. We use the probability to encounter a certain level of market impact costs on a trade to predict the bucket in which market impact costs will fall. The higher the probability that a trade will cause high trading costs, the higher the bucket we will predict for that trade.

We take the same buckets for buys and sells and define them in such a way that we have five buckets with increasing levels of market impact costs: bucket 1 (no costs, $(-\infty, 0] \mathrm{bp})$, bucket 2 ('low costs', $(0,20] \mathrm{bp})$, bucket 3 ('average costs', $(20,50] \mathrm{bp})$, bucket 4 ('high costs', $(50,80$ ] bp), bucket 5 ('severe costs', $(80, \infty)$ bp). In practice, the choice of the buckets will depend on individual investor preferences. Given $p_{0}=0$ and $p_{5}=1$, we set four critical 'probability level's' $p_{1}, p_{2}, p_{3}$, and $p_{4}$ to assign the trades to one of the five buckets. If the 'excess probability' $\mathbb{P}\left(\mathbf{C}_{B}>T \mid \mathbf{X}_{1}, \ldots, \mathbf{X}_{N}\right)$ satisfies $p_{i}<\mathbb{P}\left(\mathbf{C}_{B}>T \mid \mathbf{X}_{1}, \ldots, \mathbf{X}_{N}\right)<p_{i+1}$ we predict that a buy will fall in bucket $i+1$. We use the in-sample period to determine optimal values of the threshold level $T$ and the cut-off probabilities and set $T=80$ and $p_{1}=0.15, p_{2}=0.25$, $p_{3}=0.375$, and $p_{4}=0.55$. For sells we proceed in a similar way and set $T=80$ and $p_{1}=0.09, p_{2}=0.15, p_{3}=0.25$, and $p_{4}=0.40 .^{5}$

Again we use an expanding estimator and evaluate the quality of the bucket approach. The upper panel of Exhibit 5 displays the classification results, both in absolute numbers and in percentages. Ideally, the percentages on the diagonals of the second and fourth panel at the right-hand-side of Exhibit 5 should be as close to $100 \%$ as possible. The higher they are, the more trades are classified in the correct buckets. Misclassification occurs when off-diagonal elements in Exhibit 5 are not equal to zero. The lower panel of Exhibit 5 displays several measures related to the overall classification quality. We consider the percentage of (1) correctly classified trades, (2) trades with no or low market impact costs that are predicted to have high or severe trading costs, (3) trades with high or severe market impact costs that are predicted to have no or low trading costs, (4) seriously misclassified trades, which are defined as trades with no or low costs classified as high or severe or vice versa, and (5)

\footnotetext{
${ }^{5}$ Although it seems obvious to classify trades on the basis of expected market impact costs, this does not work well in practice since there are considerable differences between realized and forecasted market impact costs in our sample. Therefore, we use the excess probabilities for this purpose.
} 
trades misclassified two or more buckets away from the correct bucket. We compare the resulting percentages to the 'zero-skill' or 'naive' model assigning a trade to bucket $i=1, \ldots, 5$ with probability $1 / 5$. In-sample, the bucket approach applied to buy trades strongly outperforms the zero-skill model on four out of five criteria. Out-of-sample, the bucket approach outperforms on all five criteria. In particular, the important category of trades with high or severe trading costs that are wrongly classified as having no or low costs is only $18 \%$ (versus $40 \%$ in the zero-skill model). For sells, the bucket approach again outperforms the naive model on all criteria. In line with expectations, the outperformance of the bucket approach relative to the naive model is less convincing in the out-of-sample period. However, the percentage of trades with no or low trading costs that are erroneously predicted to have high or severe costs is substantially lower than in the zero-skill model (23\% versus $40 \%$ ).

\section{Trade identification}

Another way of dealing with future market impact costs is to identify trades that have a high chance of being (too) expensive. That is, we assume that a trade is identified as expensive when the excess probability exceeds a certain critical level; i.e. when

$$
\mathbb{P}\left(\mathbf{C}^{B}>T \mid \mathbf{X}_{1}, \ldots, \mathbf{X}_{N}\right) \geq p
$$

for certain investor-specific values of the critical level $T$ and probability $p(0 \leq p \leq$ 1 ). The difference between this method and the previous two approaches is that we do not longer forecast a level or range of market impact costs for each trade, but only identify those that are likely to be expensive.

We can easily calculate the excess probability (5) for the regression model in equation (3), provided that we know the distribution of the error term. If we denote the distribution function of the noise term by $F(x)=P(\varepsilon \leq x)$, the excess probability for buys according to model 3 writes as

$$
\mathbb{P}\left(\mathbf{C}^{B}>T \mid \mathbf{X}_{1}, \ldots, \mathbf{X}_{\mathbf{N}}\right)=1-F\left(T-\beta_{0}^{B}+\sum_{j=1}^{N} \beta_{j}^{B} \mathbf{X}_{j}\right)
$$

The distribution of the noise term has to be known in advance to calculate this probability. As usual, the assumption of normality seems obvious and convenient, but is nevertheless likely to be restrictive. Therefore, we take the empirical distribution of the noise term based on the in-sample period, which avoids any parametric assumptions. This means that we calculate the probability in expression (6) as the fraction of trades in the in-sample period for which the residuals ${ }^{6}$ exceed the value in the parentheses of $F(\cdot)$ in expression (6).

\footnotetext{
${ }^{6}$ The residuals are defined as $e^{B}=\mathbf{C}^{B}-\hat{\beta}_{0}^{B}-\sum_{j=1}^{N} \hat{\beta}_{j}^{B} \mathbf{X}_{j}$.
} 
To assess the accuracy of the trade identification procedure, we do not focus on single values of $T$ and $p$, but instead consider a range of critical levels $p=$ $0,0.05, \ldots, 1$ and $T=0,10, \ldots, 200$ and count all correctly identified trades in the out-of-sample period. That is, we count all buy trades $i$ for which $\mathbb{P}\left(\mathbf{C}_{i}^{B}>T \mid\right.$ $\left.\mathbf{X}_{1}, \ldots, \mathbf{X}_{N}\right) \geq p$ and, at the same time, $\mathbf{C}_{i}^{B}>T$. In practice, investors will focus on a smaller area of interest in Exhibits 6 (a) and (b) on the basis of their preferences and experience.

The heat maps in Exhibits 6 (a) and (b) depict the number of correctly identified buys and sells as percentage of the total number of trades identified for the whole range of critical values. Although the classification is more accurate for higher values of the critical level, there is a trade-off between the accuracy of the method and the number of trades selected. The number of trades with trading costs exceeding $T$ bp is small for high values of $T$, making the reported percentages in these areas somewhat suggestive.

To illustrate the use of the heat maps, we consider the one for buys in Exhibit 6 (a). When we select all buys $i$ for which $\mathbb{P}\left(\mathbf{C}_{i}^{B}>150 \mid \mathbf{X}_{1}, \ldots, \mathbf{X}_{N}\right) \geq 0.3$, the number of correctly identified trades equals $33 \%$ (9 out of 27 ) and thus falls in the category $30-40 \%$. This means that $33 \%$ of the buy trades identified do, in fact, have market impact costs in excess of $150 \mathrm{bp}$.

\section{CONCLUSIONS}

When a relatively small group of equity trades causes the major part of the market impact costs of an investment portfolio, a reduction of the trading costs of comparatively few expensive trades would already result in substantial savings on total trading costs. For the equity trades analyzed in this paper, executed by the world's second largest pension fund, we find that only $10 \%$ of all trades determines $75 \%$ of total market impact costs. Simulations confirm that there is a nonlinear tradeoff between trading costs and the number of trades executed.

Since trading cost depend to some extent on controllable factors such as broker intermediation, trade timing, and trading venue, investors can try to reduce trading costs by carefully controlling these factors. As a first step in this direction, this paper has discussed various methods to obtain forecasts of market impact costs that can help to identify potentially expensive trades. Applied to the global equity trades of the world's second largest pension fund in the world in the first quarter of 2002, the proposed methods succeed in identifying a reasonable number of expensive trades. The results illustrate the productive role that model-based forecasts can play in trading cost management.

\section{ACKNOWLEDGEMENTS}

The authors would like to thank the participants of the research seminars at De Nederlandsche Bank, University of Twente, and Delft University of Technology. The usual disclaimer applies. The views expressed in this paper are not necessarily 
shared by DNB and ABP Investments or its subsidiaries.

\section{REFERENCES}

Bikker, J.A., L. Spierdijk, and P.J. van der Sluis. "Market Impact Costs of Institutional Equity Trades". Forthcoming in Journal of International Money and Finance (2007).

Chan, L.K.C. and J. Lakonishok. "The Behavior of Stock Prices around Institutional Trades". Journal of Finance, 50 (1995), pp. 1147-1174.

Chan, L.K.C. and J. Lakonishok. "Institutional Equity Trading Costs: NYSE versus Nasdaq". Journal of Finance, 52 (1997), pp. 713-735.

Cheng, M. "Trading Costs Analysis and Management". Equity Trading: Execution and Analysis (2003), pp. 26-34.

Keim, D.B. and A. Madhavan. "The Cost of Institutional Equity Trades". Financial Analysts Journal, 54 (1998), pp. 50-69.

Konstance, M.S. "Pretrade Cost Analysis and Management of Implementation Shortfall". Equity Trading: Execution and Analysis (2003), pp. 8-12.

Wagner, W.H. and M. Edwards. "Best Execution". Financial Analysts Journal, 49 (1993), pp. 65-71. 


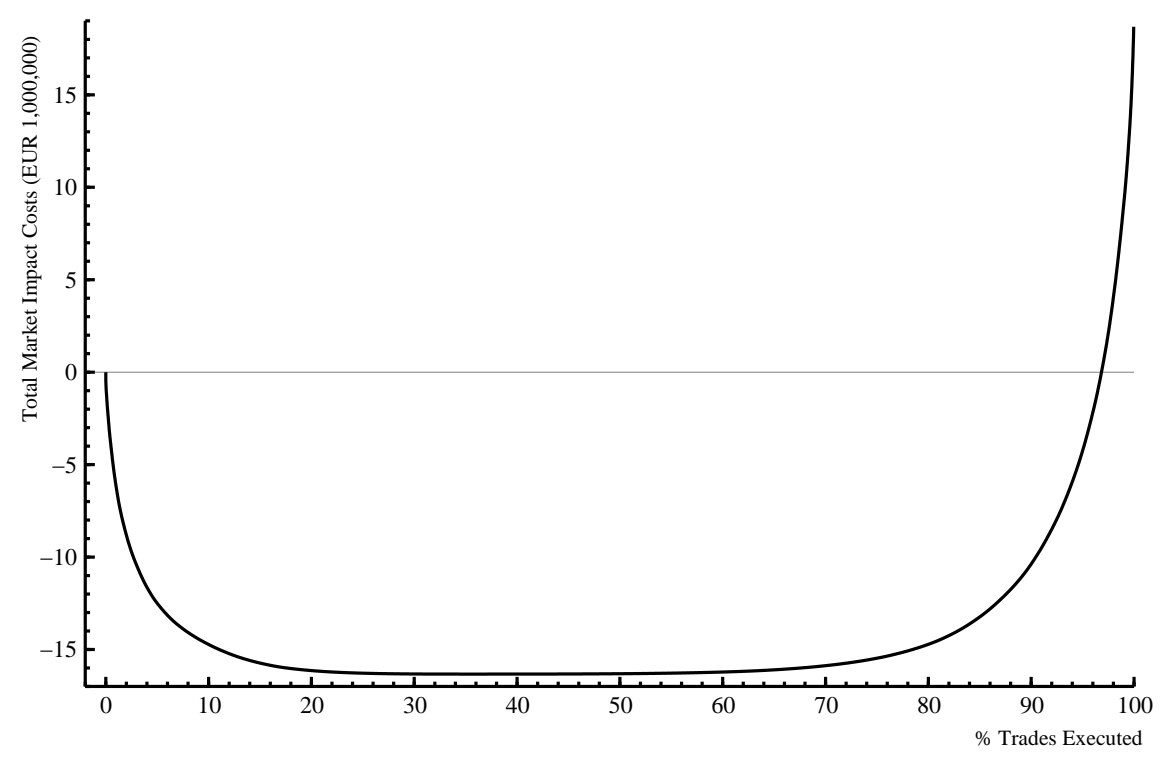

EXHIBIT 1: TRADES AND THEIR CONTRIBUTION TO TOTAL MARKET IMPACT COSTS

This exhibit displays total market impact costs plus commission (in Euro) as a function of the percentage of trades executed. For example, when only the $97 \%$ cheapest trades are executed, no trading costs are incurred. 
(a) 3D plot

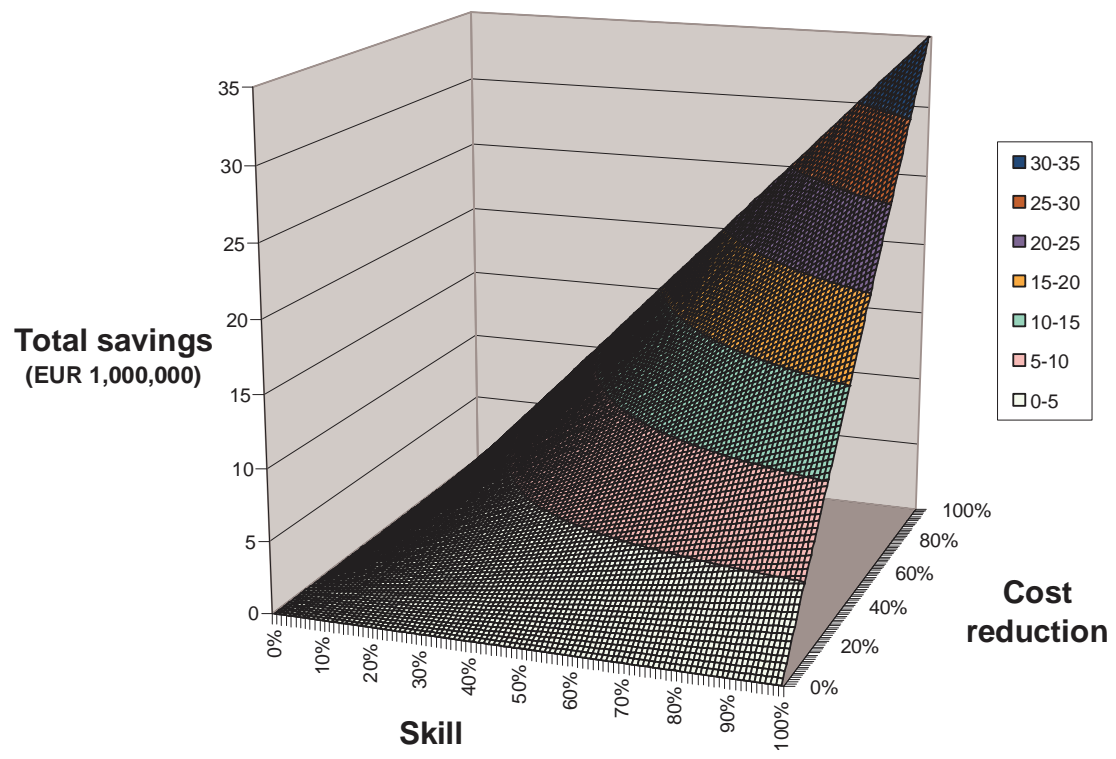

(b) Contour plot for various levels of total savings

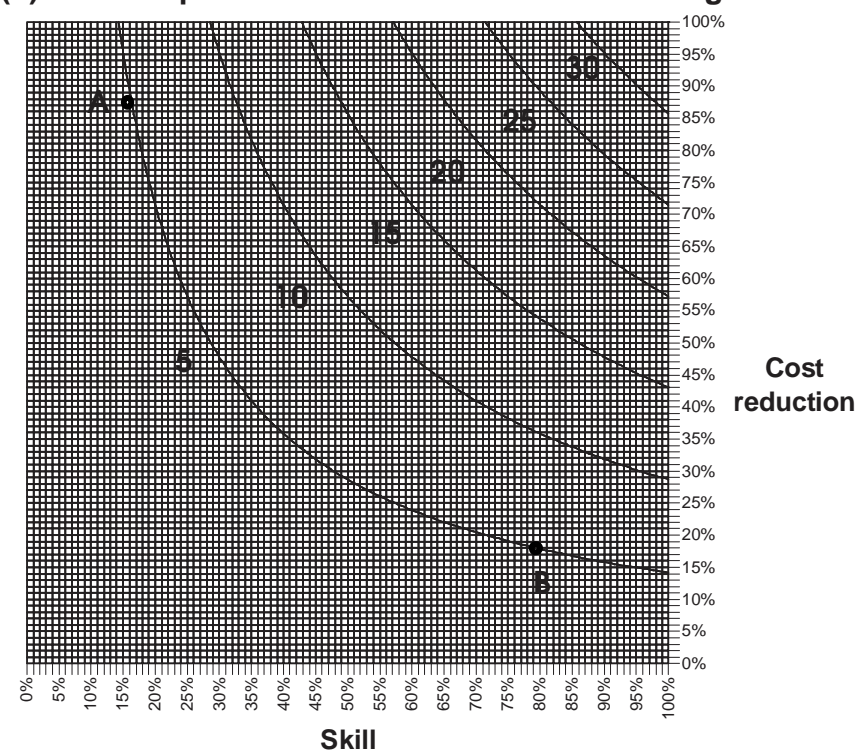

EXHIBIT 2: RELATION BETWEEN INVESTOR SKILLS, COST REDUCTION, AND SAVINGS

Exhibit (a) shows total (expected) savings on market impact costs including commission (in Euro) for each percentage of investor skills and cost reduction per trade. Exhibit (b) displays the corresponding contour plot for various levels of total savings. The total savings on trading costs are displayed at each contour line (in million Euro). 
variable

const

momentumperc

tradesizertdv

marketcap

agencysingledum

quantdum

preopendum

morningdum

Wednesdaydum
Fridaydum earlymonthdum

Jandum

Febdum

NYSEdum

Nasdaqdum

$R^{2}$ 5-day volume-weighted average return prior to trading (in \%) trade size relative to 30-day average daily volume prior to trading (in \%) (log) market capitalization 3 months prior to trading (in billion Euro)

$0 / 1$ variable for agency/single (1) and principal (0) trades

$0 / 1$ variable for trades executed by quantitative (1) and fundamental (0) funds

$0 / 1$ variable for trades sent to broker during pre-opening of the market

$0 / 1$ variable for trades sent to broker in the morning (after pre-opening)

$0 / 1$ variable for trades executed on Wednesday

$0 / 1$ variable for trades executed on Thursday

$0 / 1$ variable for trades executed on Friday

$0 / 1$ variable for trades executed at the beginning of the month

$0 / 1$ variable for trades executed in January

0/1 variable for trades executed in February

$0 / 1$ variable for trades executed on NYSE

$0 / 1$ variable for trades executed on Nasdaq estimate

$-27.09$

t-value

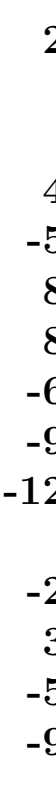$$
-12
$$

23.92
6.00
3.41
49.41
-55.95
82.25
86.70
-69.08
-90.93
-127.40
12.59
-21.75
34.63
-51.97
-95.95

$-1.10$

4.99

2.95

1.65

1.65
6.70

6.70
-3.74

7.75

6.81

$-9.71$

$-7.02$

$-9.30$

0.90

$-3.02$

3.10

3.10
-5.92

$-5.53$

0.18 estimate t-valu

$5.11 \quad 0.1$

-21.50 -4.8

$7.63 \quad 3.0$

$\mathbf{- 8 . 8 0}-2.6$

$-6.20-0.6$

$\mathbf{5 6 . 5 4} 3.3$

$\begin{array}{ll}-75.67 & -4.9\end{array}$

$\mathbf{- 8 9 . 1 3}-5.6$

65.18

92.02

109.95

42.90

$-8.66$

$-45.00$

66.03

139.03

$$
6.5
$$$$
5.6
$$

8.2

2.0

$-1.0$

$-3.4$

6.4

8.4

\section{EXHIBIT 3: DESCRIPTION OF DETERMINANTS OF MARKET IMPACT COSTS AND CORRESPONDING ESTI- MATION RESULTS}

This exhibit displays a list of determinants of market impact costs and their estimated coefficients (with corresponding $t$-values) based on the model of equation (3). Coefficients in bold face are significant at a $5 \%$ level. 
(a) Buys in-sample

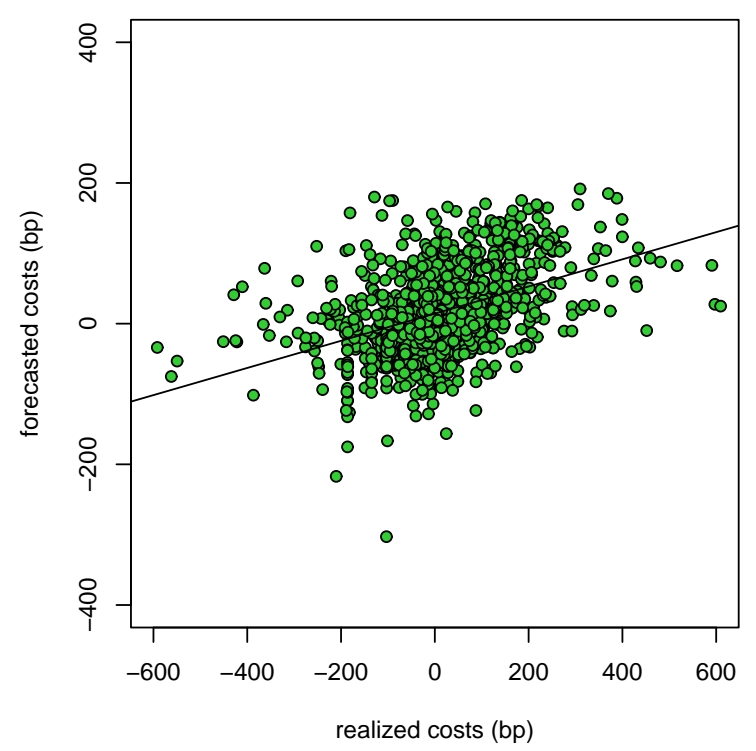

(c) Buys out-of-sample

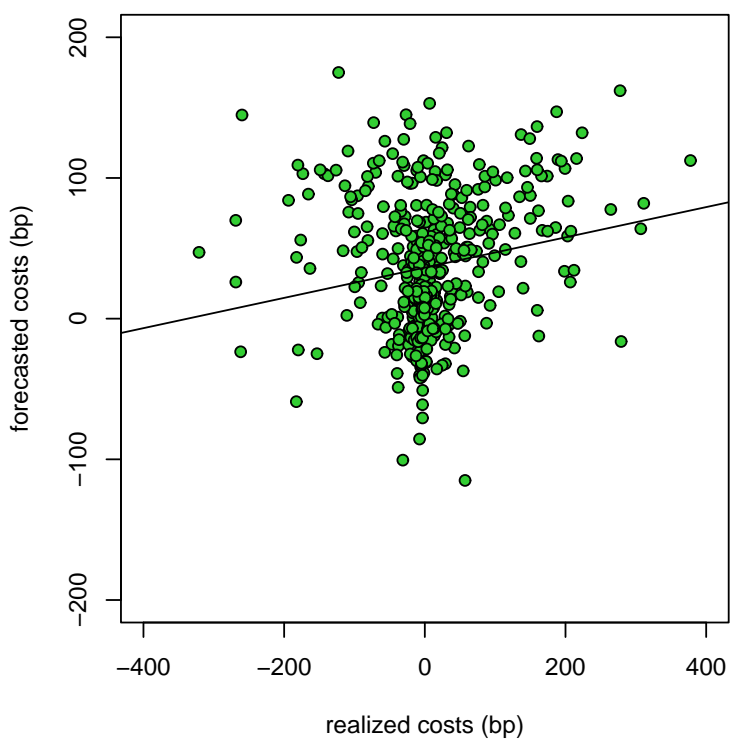

(b) Sells in-sample

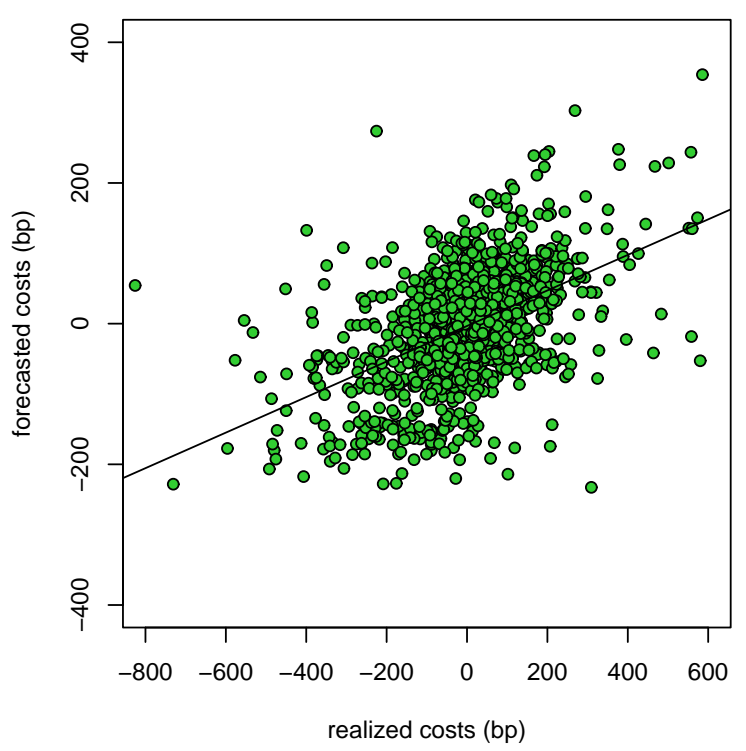

(d) Sells out-of-sample

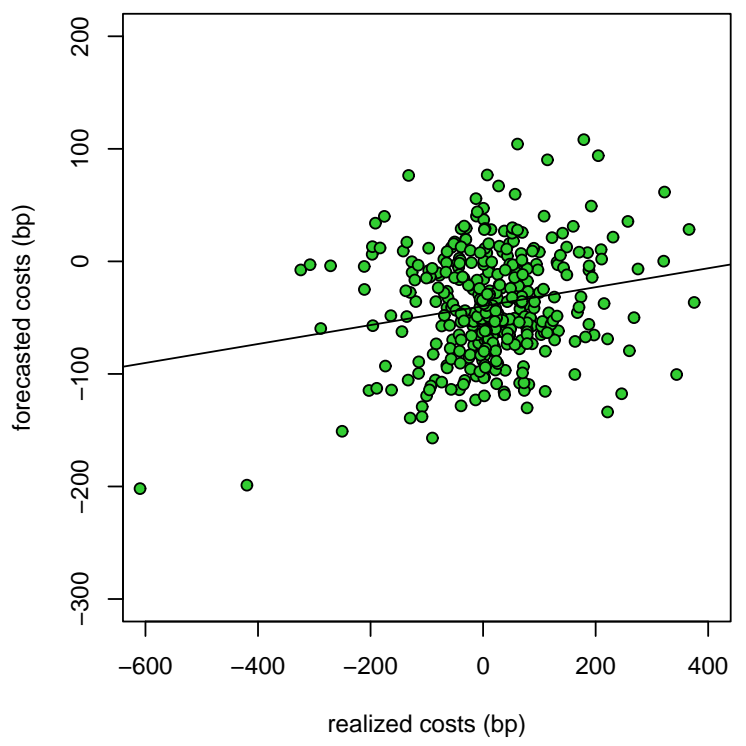

\section{EXHIBIT 4: REALIZED AND FORECASTED MARKET IMPACT COSTS}

This exhibit displays realized and forecasted market impact costs for buys and sells (both in-sample and out-of-sample), together with regression lines that express forecasted trading costs as a function of realized costs. 
in-sample

out-of-sample

\section{BUYS}

\begin{tabular}{|c|c|c|c|c|c|c|c|c|c|c|c|}
\hline$\#$ & realized & & & & & \# & realized & & & & \\
\hline predicted & 1 & 2 & 3 & 4 & 5 & predicted & 1 & 2 & 3 & 4 & 5 \\
\hline 1 & 341 & 65 & 75 & 31 & 46 & 1 & 69 & 21 & 9 & 3 & 4 \\
\hline 2 & 130 & 68 & 70 & 43 & 57 & 2 & 66 & 27 & 9 & 5 & 4 \\
\hline 3 & 78 & 43 & 64 & 33 & 69 & 3 & 47 & 18 & 11 & 8 & 7 \\
\hline 4 & 37 & 17 & 17 & 12 & 41 & 4 & 24 & 10 & 8 & 6 & 20 \\
\hline 5 & $\underline{28}$ & $\underline{8}$ & 15 & 14 & 103 & 5 & $\underline{35}$ & $\underline{6}$ & $\underline{8}$ & $\underline{5}$ & 27 \\
\hline total & $6 \overline{14}$ & $20 \overline{1}$ & $2 \overline{41}$ & $1 \overline{33}$ & $\overline{316}$ & total & $2 \overline{41}$ & $8 \overline{2}$ & $4 \overline{5}$ & $2 \overline{7}$ & $\overline{62}$ \\
\hline$\%$ & realized & & & & & $\%$ & realized & & & & \\
\hline predicted & 1 & 2 & 3 & 4 & 5 & predicted & 1 & 2 & 3 & 4 & 5 \\
\hline 1 & 55.5 & 32.3 & 31.1 & 23.3 & 14.6 & 1 & 28.6 & 25.6 & 20.0 & 11.1 & 6.5 \\
\hline 2 & 21.2 & 33.8 & 29.0 & 32.3 & 18.0 & 2 & 27.4 & 32.9 & 20.0 & 18.5 & 6.5 \\
\hline 3 & 12.7 & 21.4 & 26.6 & 24.8 & 21.8 & 3 & 19.5 & 22.0 & 24.4 & 29.6 & 11.3 \\
\hline 4 & 6.0 & 8.5 & 7.1 & 9.0 & 13.0 & 4 & 10.0 & 12.2 & 17.8 & 22.2 & 32.3 \\
\hline 5 & $\underline{4.6}$ & $\underline{4.0}$ & $\underline{6.2}$ & $\underline{10.5}$ & 32.6 & 5 & 14.5 & $\underline{7.3}$ & $\underline{17.8}$ & $\underline{18.5}$ & $\underline{43.5}$ \\
\hline total & 100 & 100 & 100 & 100 & 100 & total & 100 & $\overline{100}$ & 100 & $\overline{100}$ & $\overline{100}$ \\
\hline
\end{tabular}

SELLS

$\begin{array}{lrrrrrr}\# & \text { realized } & & & & & \\ \text { predicted } & \mathbf{1} & \mathbf{2} & \mathbf{3} & \mathbf{4} & \mathbf{5} & \text { predicted } \\ \mathbf{1} & 198 & 11 & 8 & 7 & 12 & \\ \mathbf{2} & 188 & 49 & 24 & 21 & 36 & \\ \mathbf{3} & 175 & 64 & 36 & 18 & 46 & \\ \mathbf{4} & 81 & 33 & 31 & 26 & 67 & \\ \mathbf{5} & \underline{52} & \underline{12} & \underline{36} & \underline{21} & \underline{121} & \\ \text { total } & 694 & 169 & 135 & 93 & 282 & \text { total }\end{array}$

ealized

$\begin{array}{rrrrr}\mathbf{1} & \mathbf{2} & \mathbf{3} & \mathbf{4} & \mathbf{5} \\ 30 & 2 & 6 & 6 & 6 \\ 32 & 15 & 17 & 12 & 23 \\ 58 & 29 & 17 & 19 & 15 \\ 30 & 8 & 7 & 9 & 19 \\ \underline{8} & \underline{4} & \underline{1} & \underline{5} & \underline{8} \\ 158 & 58 & 48 & 51 & 71\end{array}$

$\begin{array}{lrrrrr} & \text { realized } & & & & \\ \text { predicted } & \mathbf{1} & \mathbf{2} & \mathbf{3} & \mathbf{4} & \mathbf{5} \\ \mathbf{1} & 28.5 & 6.5 & 5.9 & 7.5 & 4.3 \\ \mathbf{2} & 27.1 & 29.0 & 17.8 & 22.6 & 12.8 \\ \mathbf{3} & 25.2 & 37.9 & 26.7 & 19.4 & 16.3 \\ \mathbf{4} & 11.7 & 19.5 & 23.0 & 28.0 & 23.8 \\ \mathbf{5} & \underline{7.5} & \underline{7.1} & \underline{26.7} & \underline{22.6} & \underline{42.9} \\ \text { total } & 100 & 100 & 100 & 100 & 100\end{array}$

predicted

realized

$\begin{array}{rrrrr}\mathbf{1} & \mathbf{2} & \mathbf{3} & \mathbf{4} & \mathbf{5} \\ 19.0 & 3.4 & 12.5 & 11.8 & 8.5 \\ 20.3 & 25.9 & 35.4 & 23.5 & 32.4 \\ 36.7 & 50.0 & 35.4 & 37.3 & 21.1 \\ 19.0 & 13.8 & 14.6 & 17.6 & 26.8 \\ \underline{5.1} & \underline{6.9} & \underline{2.1} & \underline{9.8} & \underline{11.3} \\ 100 & 100 & 100 & 100 & 100\end{array}$

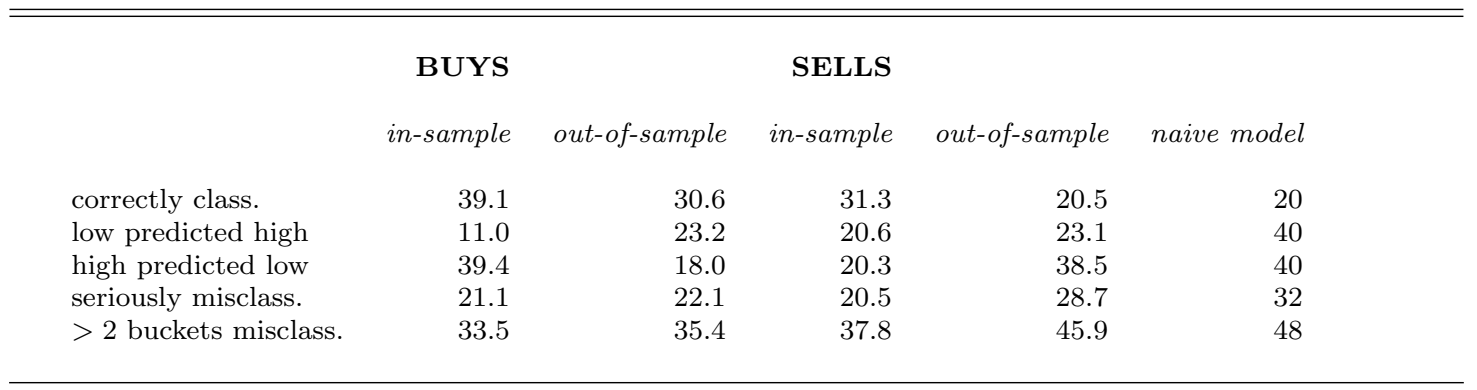

\section{EXHIBIT 5: CLASSIFICATION RESULTS BASED ON BUCKET APPROACH}

This upper panel of this exhibit displays the classification results for the bucket approach. For both buys and sells it reports the numbers and percentages of trades with realized costs in bucket $i$ and forecasted bucket $j(i, j=1,2,3,4,5)$. The lower panel reports several quality measures corresponding to the bucket classification method. 


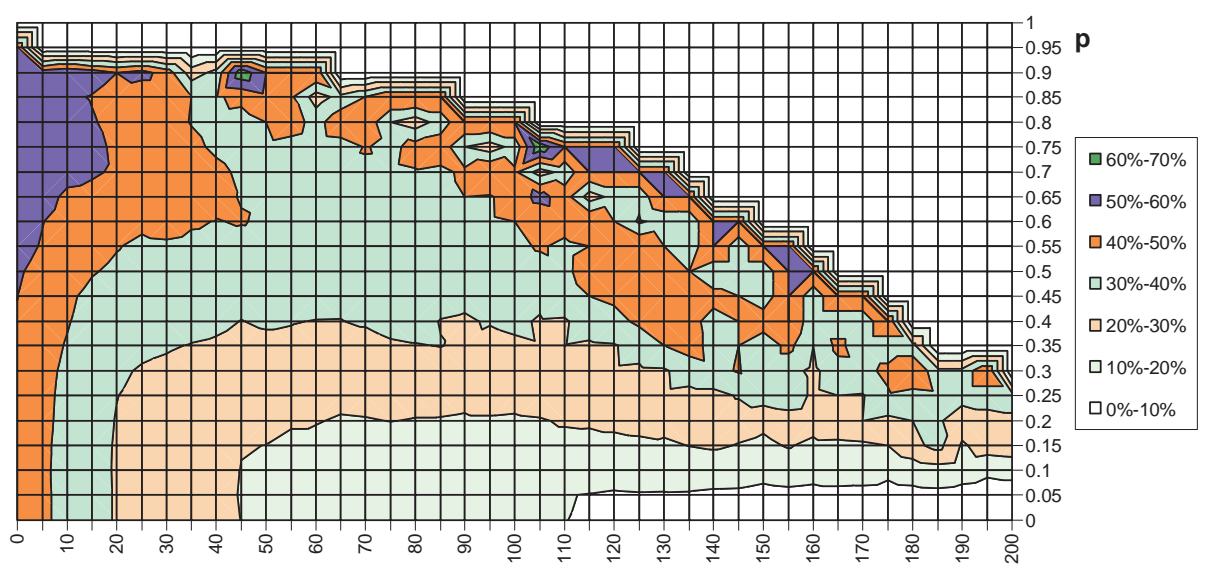

$\mathbf{T}$

(b) Percentage of correctly identified sells

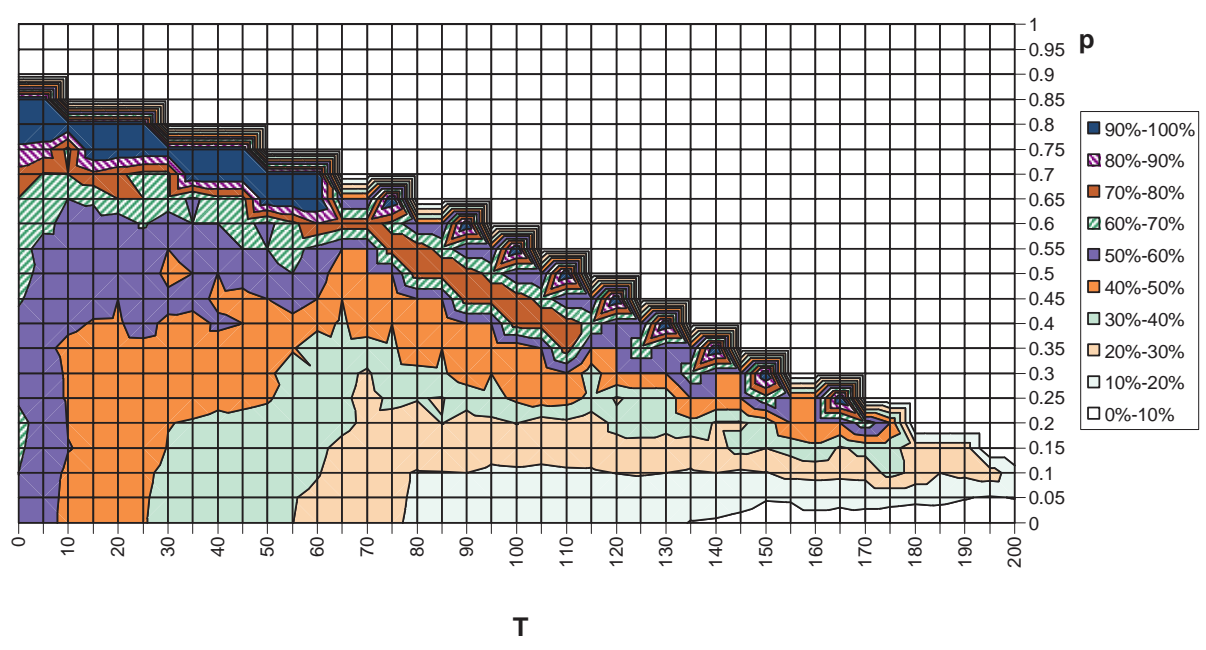

EXHIBIT 6: HEAT MAPS FOR OUT-OF-SAMPLE PERIOD

This exhibit shows heat maps indicating the percentages of correctly classified buys and sells, respectively, based on the trade identification method. The colors in the heat map indicate the percentages of correctly classified trades for a range of critical levels $T$ (horizontal axis) and probabilities $p$ (vertical axis). 